\title{
Ethics in the digital age
}

\section{A ética na era digital}

\author{
Paulo Roberto lima Carreiro, TCBC-MG ${ }^{1}$
}

"... Whatsoever I shall see or hear in the course of my profession, as well as outside my profession in my intercourse with men, if it be what should not be published abroad, I will never divulge, holding such things to be holy secrets." (Hippocrates, around 400 B.C.).

Sinem ince the Hippocratic era there has been concern with medical secrecy and respect for confidentiality, and consequently the patients' autonomy. The essence of this principle, however, in light of new contexts and new technologies, rise some necessary ethical reflections, among them the misuse of images (photos and / or videos) of patients.

Digital technology has developed rapidly in recent years, in a way that today it is very simple to capture and store images in increasingly portable devices. It is common sight in hospitals today, doctors, nurses, residents and students recording calls to patients or "interesting cases" on their cameras or cell phones, with no embarrassment and, in almost all cases, without due consent. On the other hand, there is no regulation or oversight by institutions, creating situations that can be harmful not only to patients but also to those responsible for obtaining or disseminating images.

The medical documentation through pictures or videos can bring us many benefits, including: preparation of texts, lectures and multimedia presentations for educational and training purposes; scientific documentation for evolutionary monitoring or research; use of images in telemedicine, allowing an expert to give an opinion remotely; use in forensic medicine. However, the improper use or storage of the images may result in a hazardous situations for the patients, primarily through undue exposure of their images and breaches of confidentiality.

The images obtained in a medical setting belong to the patient and thus requires the use of explicit consent. In situations where the image does not allow the identification of the patient (eg, picture of a lesion, ultrasound image, X-rays, CT scans), this authorization is not required. However, some magazines and periodicals recommend, or even require, in the case of article publication, obtaining the patient's consent.
In elective situations, consent is requested more often because, in most cases, there is a relationship between the physician and the patient, allowing the doctor to explain in detail the objectives of the request. This is a frequent practice in plastic surgery, when surgeons document the "before" and "after" to assess the results of the procedure. An important issue is that, in any event, these photos should under no circumstance be used for the purpose of publicity favoring the surgeon. Several consent protocols elaborated by Plastic Surgery societies are available for this purpose.

In emergency care services, due to the characteristics of these environments, undue imaging is observed more frequently. In these situations, often the patient is not able to express his/her consent for a number of reasons: being unconscious or under the influence of drugs or medications, intubation or airway prosthesis, being in critical condition, physical or psychological stress, a fragile situation, etc. In addition to these factors, it is difficult to maintain an efficient monitoring scheme 24 hours a day in a place where a large number of people wander, with different levels of information. These difficulties are also present in the pre-hospital environment, where anyone with a camera or cell phone can record images of the scene. Portraying real and shocking scenes, such images are frequently and indiscriminately uploaded to internet sites, unfortunately often by people involved in the pre-hospital care (doctors, paramedics, police, firefighters).

If we assume that a photo of the patient obtained during his hospitalization for medical documentation is a (photographic) record that is part of the medical record, to download the photo file to a personal computer or any other media would be improperly copying or take possession of part of the medical records, since we could only do so with permission of the patient.

Another important issue is how to store images securely. Once the use of images is authorized, they should preferably be stored with software specifically for this purpose and have security codes, allowing access only to authorized staff. The storage in CDs, flash drives, laptops or on cameras and cell phones is extremely insecure, as these images will eventually be intentionally or accidentally shared. Furthermore, these devices are subject to loss or theft.

John XXIII Hospital - FHEMIG (Foundation Hospital of Minas Gerais); UNIFENAS Faculty of Medical Sciences, Belo Horizonte, Minas Gerais State - MG Brazil. 
Paradoxically, not only the patients are the ones who are exposed to the misuse of imaging resources. Health professionals and even institutions can also be victims of this practice. Relatives or can register images of attendances, environments or events that occur in the hospital and then use them in legal questions or complaints in the media.

Given the above, some measures should be considered in order to protect patients from undue exposure, and professionals and institutions from litigious situations. Initially the most important measure would be the awareness and education of health professionals, residents and students in relation to the patient $s^{\prime}$ fundamental rights. Other measures would be the development of institutional protocols for imaging and effective monitoring of its implementation.

Despite the continuous evolution, the inevitable changes and the incorporation of new technologies, the observance of basic rights of patients is immutable and should guide the conduct of all professionals involved directly or indirectly in patient care. 